\title{
China's Economic Situation, Response and Prospect under the Epidemic Situation
}

\author{
Lan Guo \\ Xianda College of Economics and Humanities, Shanghai International Studies University, \\ Shanghai, China \\ 520201787@qq.com
}

\begin{abstract}
On the basis of the demand side and the supply side, the paper respectively analyzed how novel Coronavirus affected Chinese economy: the weak demand caused the demand curve to move to the left, equilibrium output decreased and equilibrium price decreased. Due to the reduction of labor costs, enterprises have the incentive to increase production capacity at the production end. However, when the price is lower than the average variable cost of enterprises, the number of bankruptcies in the society starts to increase. One of the immediate causes of the economic downturn caused by COVID-19, which led to GDP growth of $-6.8 \%$ in the first quarter of 2020 , is a lack of consumption. Therefore, it is suggested that the government can appropriately reduce consumption tax and issue consumption vouchers, to improve consumer confidence and increase output. The ARIMA model was used to predict economic growth for the next seven quarters.
\end{abstract}

Keywords: Supply; Demand; Vouchers; Policy; ARIMA; Economic Forecasting; Keynesian-ism.

\section{Introduction}

Novel Coronavirus epidemic broke out in China at the beginning of 2020. Due to insufficient understanding of virus and rapid flow of people, the virus spread rapidly. The rapid outbreak of the epidemic led the government to restrict social mobility, which directly affected domestic consumption. The pessimistic social expectations led to the decline in investment, and thus the decline in GDP.

\section{The Current State of China's Economy and its Causes}

\subsection{Internal Analysis}

Due to the rapid outbreak of coVID-19, people have been staying at home. The decline in social mobility has hit China's domestic consumer industry first. The industries seriously affected by price comparison are catering, tourism, accommodation, aviation, transportation and retail. These industries rely heavily on social mobility. The collapse in social mobility, based on the lethality of these industries, and the rapid decline in revenues, has made life difficult for firms in these industries. Workers returned to the city, factory resumption was delayed, enterprises stopped production to reduce production, and investment in manufacturing, real estate, and infrastructure was basically stagnant in the short term. The decline in demand leads to the decline in prices and the decline in corporate profits. When prices are lower than the average variable cost of enterprises, the number of bankruptcies of sums begins to increase. The decline in household consumption will be slowly transmitted to the production end. As enterprises expect to consume less in the future, they reduce production to avoid the increase in costs caused by inventory overhang.

\subsection{External Analysis}

Despite the global economic downturn in 2019 and the delayed Sino-U.S. trade war, China's foreign trade imports and exports still have a bright performance: the total value of imports and exports exceeded 31.5 trillion yuan, an increase of 3.4\% year-on-year, and still maintained global goods The status of the largest trading country. Entering 2020, the world economy is still sluggish and external demand is weakening. In this case, this new coronary pneumonia epidemic will make it difficult to expand exports. Even if the epidemic does not result in no one asking for Chinese goods, 
the epidemic will increase the cost of exporting goods. The commodities that have been produced are now exported to foreign countries, and the time for customs clearance and quarantine needs to be increased. This will increase transportation costs, quarantine costs and warehouse costs. The total cost of commodities will increase, which will lead to a decline in competitiveness. However, the cost increase caused by quarantine will not be particularly large, and its impact may not even be as great as the impact of tariffs. But in the medium term, in order to control the spread of the epidemic, the factory cannot resume work during this time, and production Will stagnate, which will lead to out of stock for a while. For many foreign trade companies, the migration of suppliers and customers brought about by out of stock will be a relatively big challenge in the medium term. Major economies in the world have been seriously affected by the epidemic. Consequently, international demand has declined and China's exports have declined .Chinese factories overseas will be adversely affected by shutdowns; Due to the unstable supply chain and logistics caused by the epidemic overseas, relevant important materials in China cannot be supplied in time, which leads to the overstocking of factories and rising storage costs.

Based on the above analysis, the decline of household income leads to the sluggish internal demand, while the severe outbreak of foreign epidemic leads to the decline of external imports. Overall, demand is insufficient and the aggregate demand curve moves to the left, reducing equilibrium output.

\section{Analysis of Economic Automatic Stabilizer}

When demand drops, AD curve moves to the left, equilibrium output drops, price level drops, employee wage level drops, and then the production cost of enterprises drops. Therefore, AS curve moves to the right, equilibrium output increases, until output returns to the natural output level, AS curve stops moving, and the final price level becomes lower.

\section{Solutions}

\subsection{Loose Monetary Policy and Expansionary Fiscal Policy}

Loose monetary policy increases the money supply, which IS shown in the IS-LM model as LM curve moves to the right. When IS and LM re-reach equilibrium, output increases and interest rate decreases. Expansionary fiscal policy moves the IS curve to the right, increasing equilibrium output and raising interest rates.

\subsection{Issue of Consumption Vouchers}

Issuing consumption coupons can enrich market confidence, increase residents' willingness to consume, and boost the economy through consumption, thus increasing employment.

Direct discovery of gold cannot effectively promote consumption, but will also produce a great crowding out effect, income effect is not obvious, and direct discovery of gold may increase social panic, making consumer sentiment more conservative. However, the issuance of shopping vouchers is one of the best ways to promote consumption, which is also a form of price discrimination for businesses, and can help alleviate poverty with targeted measures .For the rich, the time cost to obtain the consumption voucher is relatively high, and the cost is greater than the benefit, so the rich are more inclined not to receive the consumption voucher.

\subsubsection{Consumption Voucher Type}

Consumption voucher is a special voucher which can replace cash and is a tool to adjust the economy. When the economic recession causes a sharp decline in private consumption power, the government issues consumption vouchers as payment vouchers for people's consumption, hoping to stimulate consumption by enhancing people's purchasing power and consumption desire, thus driving the growth of production and investment and driving the economic recovery. The vouchers will have a definite expiry date, and a shorter one. 
The type of vouchers to be issued depends on the severity of the epidemic. Industries that used to rely on strong passenger flows have been severely affected by the rapid spread of the epidemic in 2020, as countries and regions have restricted the movement of people. For example, catering industry, transportation industry, tourism industry, film industry, accommodation industry and so on have been hit more. Therefore, the social issue of food vouchers, travel vouchers, travel vouchers, film vouchers and accommodation vouchers account for more.

\subsubsection{The Main Body of Issuing Consumption Coupons}
a. The government.
b. The enterprise shall distribute the products by itself
c. Jointly issued by the government and the industry or enterprise

\subsubsection{Analysis of the Function of Consumption Coupons}

The issuance of consumption coupons can increase residents' buying willingness and increase social demand. The exuberant social demand is transmitted to enterprises and promotes enterprises to expand reproduction. In this way, the employment demand of enterprises will increase, the unemployment rate will decrease, output will increase, workers' wages will rise, and consumption will increase.

(1) Multiplier effect, through the consumption of vouchers can leverage a greater social turnover.

Using the term of consumption voucher can increase consumption in a short time, alleviate the economic downturn, and thus build market confidence.

(2) Increase the speed of money flow to ease the pressure on the capital end of enterprises.

During the epidemic, businesses shut down, inventories of goods overstocked, and business costs kept rising. It can effectively reduce the inventory cost, accelerate the capital flow and improve the confidence of enterprises.

(3) Leave a window of time for further policy development.

According to Keynes, when an economic crisis occurs, the government will build a lot of infrastructure, which in turn will play a role in boosting the economy .However, infrastructure construction cannot immediately improve the economic fundamentals. There is a process from project approval to construction to economic stimulation. And until the investment pull kicks in, the economy is in a downward spiral. In this time gap, coupled with the short-term policy of consumption voucher, it can prevent the economic transition from falling, reduce the impact of economic crisis on a country's economy, and lay a good foundation for the play of investment-driven role.

(4) Social wealth is being distributed and social equity promoted

The recipients of consumption vouchers are mostly people with poor economic conditions. The epidemic has led to work stoppages and reduced income.. The consumption voucher can be used as a supplement to the consumption power, so that the low-income people can get certain security and benefits. In addition, poor people are more sensitive to price, so when the price of goods falls relatively, they will buy more and increase the income of enterprises.

\subsubsection{Impact}

Short-term impact: In the short term, through the multiplier effect, consumption bonds can effectively increase the frequency of social transactions, increase the speed of capital flow, show a certain improvement in the economy, establish market confidence, and win the time window for the government to make the next economic policies.

Long-term impact: Since consumption coupons are not sustainable in the long term, most shortterm consumers prefer to use them to buy daily necessities, durable goods and other products with a long shelf life. Therefore, consumption coupons only play the role of inter-term consumption, but cannot play an effective and sustainable role in the economy. At the same time, consumption voucher will destroy the structure of market economy, make some industries or enterprises that are not in harmony with social development continue to survive in the market, deteriorate the structure of market economy, and reduce the efficiency of market allocation of resources. 
Conclusion: Consumption voucher can only be used in the short term. In the medium and long term, the overall disadvantages outweigh the advantages. In the long run, if consumption is to be truly driven, we need to further adjust the structure and distribution of national income, improve the income distribution system as soon as possible, and increase the share of personal income in total national income.

\section{The Economic Outlook for the Future}

This paper selects the GDP of the first quarter of 2006 - the first quarter of 2020 and the year-onyear economic growth rate for research and analysis. The visualization trend of relevant indicators is shown in the following figure:

According to the figure above, we can see that the economic growth in the past 15 years has been relatively stable and basically maintained above 6 . However, due to the coVID-19 outbreak in early 2020 , the national economy has experienced negative growth for the first time in recent 15 years. The ARIMA model was subsequently established to predict China's future macroeconomic trends and quantify the impact of coVID-19 on economic development in the first quarter of 2020.

For the time series model, we use the model to predict and analyze, and give the corresponding forecast error. The time series method is a prediction method based on deterministic mathematical expressions, which has the advantages of small calculation amount and fast speed.

The main modeling steps for differential sequences are as follows:

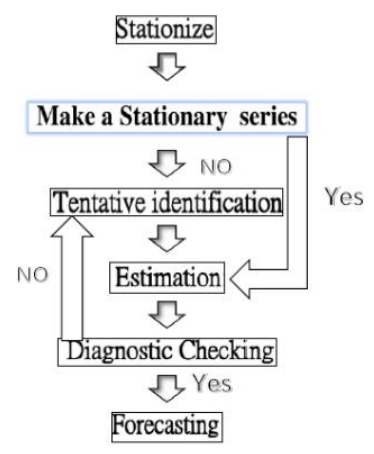

Fig 1. The auto-correlation and auto-correlation graphs of the original economic growth rate time series are drawn

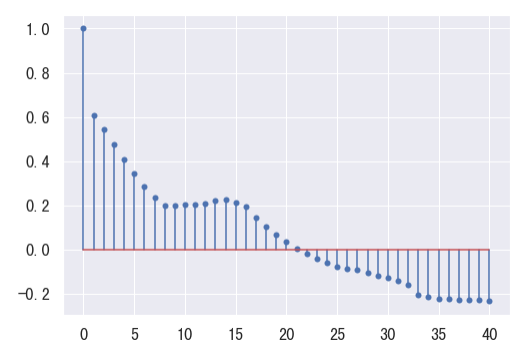

Fig 2. Original auto-correlation

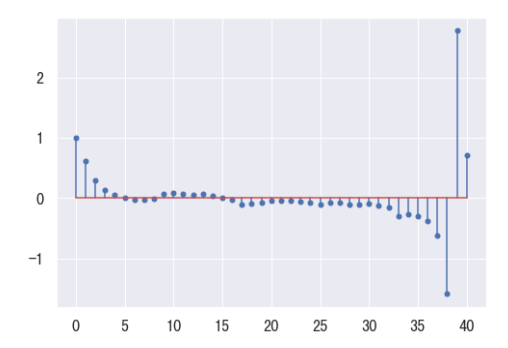

Fig 3. Original partial auto-correlation 
According to the figure above, we can see that the 0 order auto correlation coefficient and the 0 order partial auto correlation coefficient are both constant 1 . in the two figures, the partial auto correlation coefficient decreases rapidly from 1 to around 0 , and then fluctuates slightly up and down the axis of 0 as the order increases, which basically meets the requirements of geostationary. However, the auto correlation coefficient varies greatly, so it is difficult to judge the specific geostationary of the original sequence. In this paper, ADF unit root test is used to verify whether the sequence is stable.

The ADF test results are shown in the table below:

Table 1. ADF test of the original sequence

\begin{tabular}{|c|c|c|c|c|}
\hline $\mathrm{T}$ & $\mathrm{P}$ & The critical value(1\%) & The critical value $(5 \%)$ & The critical value(10\%) \\
\hline-1.204 & 0.209 & -2.606 & -1.946 & -1.612 \\
\hline
\end{tabular}

The test results show that $\mathrm{P}$ value is equal to 0.209 , which is not significant at the $90 \%$ confidence level. Therefore, we believe that the original sequence conforms to the null hypothesis and is unstable. At that time, the T-statistic was small, so we believed that the sequence was approximately stable. Therefore, we subsequently used the first-order difference method for data processing.

The ADF test results of the economic growth rate time series after the first difference are obtained as follows:

Table 2. ADF test of first-order difference sequence

\begin{tabular}{|c|c|c|c|c|}
\hline $\mathrm{T}$ & $\mathrm{P}$ & The critical value (1\%) & The critical value(5\%) & The critical value(10\%) \\
\hline-2.452 & 0.013 & -2.6075 & -1.946 & -1.612 \\
\hline
\end{tabular}

$\mathrm{P}$ equals 0.013 is less than 0.05 , which is not significant at the $95 \%$ confidence level, so the Tstatistic is significant at the $95 \%$ confidence level. It also shows that the time series after the first difference basically satisfies the stationary condition. So, we use ARIMA to do the first difference and make the prediction.

For the model, the order of the model should be determined, that is, the order of $\mathrm{P}$ and $\mathrm{Q}$ should be determined. So, let's graph ACF, PACF.

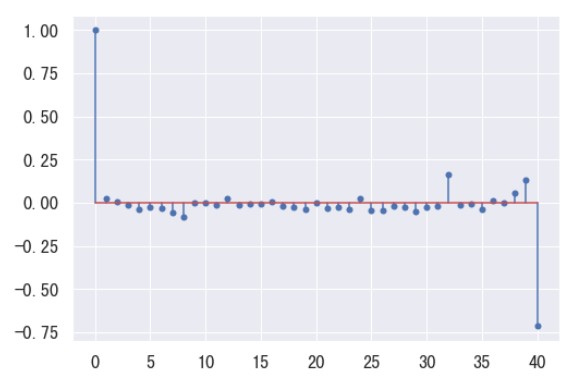

Fig 4. Auto-correlation

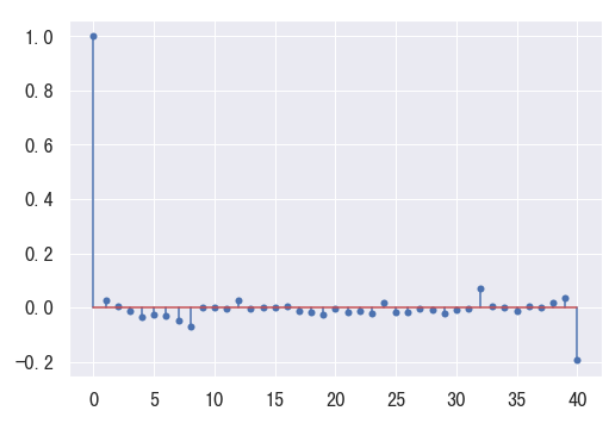

Fig 5. Partial auto correlation 
By observing the figure above, it is found that both autocorrelation and partial phase coefficients have the characteristic of trailing, and both of them have obvious first-order correlation. Therefore, we set $\mathrm{P}=1$ and $\mathrm{q}=1$. Now you can use the ARMA model for data fitting. Finally, the ARIMA $(1,1,1)$ model is constructed. The selected model parameters were used to train the time series model. Considering the huge impact of the epidemic on the economy in the first quarter of 2020, the relevant data in the first quarter of 2020 were excluded, and the previous normal economic development was used to predict the economic growth in the first quarter of 2020.

Forecast China's macroeconomic growth in the future:

Table 3. China's economic growth forecast in the future

\begin{tabular}{|c|c|c|c|}
\hline Date & Quarterly growth forecast for China & Actual value & $\begin{array}{c}\text { Quantifying the impact of the } \\
\text { epidemic }\end{array}$ \\
\hline $2020-03-31$ & 0.056861 & -0.068 & $183 \%$ \\
\hline $2020-06-30$ & 0.057231 & & \\
\hline $2020-09-30$ & 0.056685 & & \\
\hline $2020-12-31$ & 0.056071 & & \\
\hline $2021-03-31$ & 0.052376 & & \\
\hline $2021-06-30$ & 0.052525 & & \\
\hline $2020-09-30$ & 0.051904 & & \\
\hline $2021-12-31$ & 0.051223 & & \\
\hline
\end{tabular}

However, it should also be noted that due to the impact of many unknown random factors on the epidemic fluctuation, The model is not static. It is more suitable for short-term forecasting. At the same time, it is necessary to continuously add new actual data to continuously fit a forecast model that better reflects the actual situation and improve the sensitivity of the forecast. model is not immutable. It is more suitable for short-term prediction. At the same time, it needs to add new actual data constantly to fit the prediction model which can better reflect the actual situation and improve the sensitivity of prediction.

\section{Positive Factors}

The theory of National Income Measurement. (a tangible hand -- Keynesianism).

From a macro perspective, national income theory, the government has a good foundation and conditions to promote sustained economic recovery.

First, the steady recovery of the economy is obvious. Resumption of work, production, and resumption of the market are powerful and effective. The industrial service industry has achieved growth. The decline in consumer investment has continued to narrow. Market expectations are generally stable.

Second, the effects of macro policies continue to appear. This year's new burden reduction for enterprises is expected to exceed 2.5 trillion yuan, the issue of 1 trillion yuan of special anti-epidemic government bonds, the increase of 1 trillion yuan in fiscal deficit, and increased support for monetary credit. These policies will continue to support later economic recovery.

Third, the new kinetic energy continues to grow. The digital economy is accelerating overall, intelligent and technological products are growing rapidly, remote offices, online education, online consultation and other rapid expansions, new models such as unmanned retailing and live streaming have emerged, which will effectively support economic development. 


\section{Conclusion}

Through the regulation of these behaviors, the prediction of the previous model may become more biased. The growth rate of GDP will increase with the support of government policies. This is what Chinese citizens prefer to see. Hope everything goes in a good direction!

\section{References}

[1] Xiao Zhihong, Guo Mingyue. Time series analysis and SAS application [M]. Wuchang: Wuhan University Press, 2009: 145-171.

[2] Xiao Zhidong, Guo Minogue. Time series analysis and SAS application [M]. Wuchang: Wuhan University Press, 2009:145-171.

[3] He Shuyuan. Applied time series analysis [M]. Beijing: Peking University Press, 2003: 185-229.

[4] He Suyuan. Applied time series analysis [M]. Beijing: Peking University Press, 2003:185-229.

[5] Zhang Wenzeng, Ji Guoqiang, Shi Jixin, etc. Application of ARIMA model in the prediction and early warning of bacillary dysentery[J]. China Health Statistics, 2009, 26(6): 636-639.

[6] Zhang Wenz on, Ji Guoliang, Shi Jimin, et al. Application of ARIMA model in prediction and early warning of bacillary dysentery [J]. China health statistics, 2009, 26 (6): 636-639.

[7] Zhang Wentong. SPSS11 Statistical Analysis Course Advanced [M]. Beijing: Beijing Hope Electronic Press, 2002: 250-289.

[8] Zhang Wanton. SPSS11 advanced course of statistical analysis [M]. Beijing: Beijing Hope Electronic Publishing House, 2002: 250-289-Author: 85586305 links.

\section{Appendix}

import statsmodels.api as sm

import pandas as pd

import numpy as np

import matplotlib.pyplot as plt

import seaborn as sns

import matplotlib as mpl

import os

from statsmodels.stats.diagnostic import unitroot_adf

from statsmodels.tsa import stattools

\# sns.set(style="darkgrid") \#this is Seaborn's default style

mpl.style.use('seaborn')

plt.rcParams['font.sans-serif'] = ['SimHei'] \# Chinese font setting - Bold

plt.rcParams['axes.unicode_minus'] = False \# solve the problem of saving images with negative sign '-' displayed as squares

sns.set(font='SimHei',font_scale=1.5) \#solve Seaborn Chinese display problem and adjust font size

df = pd.read_excel('./data.xlsx',encoding='utf_8',sheet_name='Sheet2')

date $=$ pd.date_range('2006-3', '2020-6',freq='3M') 
Volume 13 (2021)

$\mathrm{df}[$ 'date'] $=$ date

df.set_index("date", inplace=True)

plt.stem(stattools.acf(df['year-on-year growth']));

plt.stem(stattools.pacf(df['year-on-year growth']));

df_1 = df['year-on-year growth'].diff(1)

df_1.dropna(inplace $=$ True)

unitroot_adf(df['year-on-year growth'])

unitroot_adf(df_1)

plt.figure $($ figsize $=(18,8))$

\# plt.plot(df['['absolute value '], label='GDP', color='green', linestyle='-',linewidth = '5')

plt.plot(df['year-on-year growth'], label='growth rate', color='red', linestyle='--',linewidth = '5')

\#Add legend

plt.legend()

\#Add grid

plt.grid(True)

df.iloc[0:56,:].loc[:,' ']

plt.stem(stattools.acf(df_1));\#\#autocorrelation

plt.stem(stattools.pacf(df_1));\#\#autocorrelation

def predict_plot(df,data,label):

plt.figure $($ figsize $=(18,8))$

plt.plot(df.loc[:,label], label='actual'+label,linestyle='-',linewidth = '5')

plt.axvline(x=df.index[-1], alpha=0.5, c="r", ls="--", lw=3)

\# plt.plot(test['count'], label='Test')

plt.plot(data['ARIMA'+label], label=' forecast '+label,linestyle='--',linewidth = '5')

plt.legend(loc='best')

plt.show()

fit1 = sm.tsa.statespace.SARIMAX(df.iloc[0:56,:].loc[:,','year-on-year growth '], order=(1, 1, 1), seasonal_order= $(1,1,1,4))$.fit ()

date $=$ pd.date_range('2020-3', '2022-12',freq='3M')

data $=$ pd.DataFrame $($ index $=$ date $)$ True)

data['ARIMA'+,'year-on-year growth '] = fit1.predict(start="2020-3", end="2022-12", dynamic=

predict_plot(df,data,'year-on-year growth ')

data 